\title{
Decoding Depression: Insights from Glial and Ketamine Regulation of Neuronal Burst Firing in Lateral Habenula
}

\author{
Yinul Cui, ${ }^{1,2}$ Yan Yang, ${ }^{1,2}$ YiYan Dong, ${ }^{1,2,3}$ and Hailan Hu ${ }^{1,2,3,4}$ \\ ${ }^{1}$ Center for Neuroscience and Department of Psychiatry of First Affiliated Hospital, Zhejiang University \\ School of Medicine, Hangzhou 310058, China \\ ${ }^{2}$ NHC and CAMS Key Laboratory of Medical Neurobiology, Zhejiang University, \\ Hangzhou 310058, China \\ ${ }^{3}$ Interdisciplinary Institute of Neuroscience and Technology, Qiushi Academy for Advanced Studies, \\ Zhejiang University, Hangzhou 310058, China \\ ${ }^{4}$ Mental Health Center, Zhejiang University, Hangzhou 310013, China \\ Correspondence: huhailan@zju.edu.cn
}

\begin{abstract}
The rapid antidepressant effect of ketamine is arguably one of the most significant advances in the mental health field in the last half century. However, its mechanism of action has remained elusive. Here, we describe our latest discovery on how ketamine blocks $N$-methyl-D-aspartate receptor (NMDAR)-dependent burst firing of an "antireward" center in the brain, the lateral habenula ( $\mathrm{LHb})$, to mediate its antidepressant effects. We also discuss a novel structure-function mechanism at the glia-neuron interface to account for the enhanced LHb bursting during depression. These results reveal new molecular targets for the therapeutic intervention of major depression.
\end{abstract}

According to the World Health Organization (WHO), in 2017, major depressive disorder (MDD) surpassed heart disease and cancer to become the number one disabling disease in the world. Depression is characterized by low mood, loss of motivation, feelings of despair, and an inability to feel pleasure, also known as anhedonia (Holtzheimer and Mayberg 2011; Russo and Nestler 2013; Wohleb et al. 2016). Modern views on the cause of MDD suggest that the neural activities of specific brain circuits are altered in response to external stimuli, such as stress, as a result of maladaptive molecular and cellular changes (Banasr et al. 2011; Duman and Li 2012; Wohleb et al. 2016).

The lateral habenula $(\mathrm{LHb})$, a pair of nuclei that relays information from the limbic forebrain to multiple monoamine centers, has attracted exponentially growing interest in the last 10 years (Hikosaka et al. 2008; Hikosaka 2010; Proulx et al. 2014; Hu 2016; Nuno-Perez et al. 2018; Yang et al. 2018b). A series of studies suggest that the LHb encodes negative emotions, such as disappointment and stress (Matsumoto and Hikosaka 2007; Li et al. 2011, 2013; Lammel et al. 2012; Shabel et al. 2012; Stamatakis and Stuber 2012; Warden et al. 2012; Root et al. 2014; Tian and Uchida 2015; Lecca et al. 2016; Proulx et al. 2018; Seo et al. 2018; Huang et al. 2019). The habenula may have initially escaped the attention of clinicians in brain imaging studies, perhaps because its volume is too small for the resolution of 3-tesla functional magnetic resonance imaging (fMRI). However, there is compelling evidence, from both human positron emission tomography (PET) imaging and animal autoradiography and electrophysiology studies, showing that LHb hyperactivity is a hallmark of depression (Caldecott-Hazard et al. 1988; Morris et al. 1999; Li et al. 2011, 2013). For example, in three animal models of depression, the LHb shows most significant up-regulation of metabolic activity in an unbiased whole-brain screen (Caldecott-Hazard et al. 1988).

$\mathrm{LHb}$ neurons show different patterns of spiking activity and can be divided into silent, tonic-firing, and burst-firing types (Weiss and Veh 2011). Burst firing, as a spike pattern with clusters of high-frequency action potentials (APs), provides a robust form of information coding in the central nervous system (Livingstone 1996; Lisman 1997; Harris et al. 2001; Izhikevich et al. 2003; Kepecs and Lisman 2003; Belykh et al. 2005). Bursting activities have been associated with coding of important physiological signals such as reward prediction error in the dopaminergic (DA) neurons (Schultz et al. 1997; BrombergMartin and Hikosaka 2009) and with pathological conditions such as epilepsy (Sorokin et al. 2017). However, whether bursting activity contributed to the coding of negative emotions and depressive emotion disorders remains unclear.

The discovery of the rapid antidepressant effects of ketamine represents one of the most important milestones in the psychiatric field in the last half century (Berman et al. 2000). Unlike conventional antidepressants, which often take weeks or months to show efficacy, ketamine causes rapid antidepressant effects within one hour of administration (Zarate et al. 2006; DiazGranados et al. 2010a,b; Zarate et al. 2012). Ketamine is a known blocker of $\mathrm{N}$ methyl-D-aspartate receptor (NMDAR) (Thomson et al. 1985), but the exact target of ketamine, including which

(C) 2018 Cui et al. This article is distributed under the terms of the Creative Commons Attribution-NonCommercial License, which permits reuse and redistribution, except for commercial purposes, provided that the original author and source are credited. 
brain regions and cell groups, is yet to be illuminated (Zanos et al. 2018; Cui et al. 2019). Addressing these issues and understanding ketamine actions both at the cellular and neural circuit level are crucial to unravel the core mechanism of depression and to develop new treatments with fewer side effects.

In this review, we outline our recent attempt to explore the molecular and cellular mechanisms underlying LHb hyperactivity in depression. We identified a new form of glia-neuron interaction in setting the neuronal firing mode in the LHb under a depression-like state. Furthermore, we unraveled LHb burst firing as a neural substrate of depression etiology and a crucial target of ketamine's rapid antidepressant actions. Based on these findings, we discovered the therapeutic potential of targeting the inwardly rectifying potassium channel (Kir4.1), NMDAR, and lowvoltage-sensitive T-type calcium channels (T-VSCCs) in the $\mathrm{LHb}$ for treating major depression.

\section{UP-REGULATION OF ASTROGLIAL Kir4.1 CHANNEL IN DEPRESSION-LIKE ANIMALS}

To explore the molecular mechanism underlying $\mathrm{LHb}$ hyperactivity in depression, we used an unbiased, highthroughput, quantitative proteomic screen to compare habenular protein expression between congenitally learned helpless (cLH) (Henn and Vollmayr 2005) and wild-type Sprague-Dawley (SD) rats (Li et al. 2013; Cui et al. 2018).
We identified eight up-regulated and four down-regulated candidate proteins (Fig. 1A). One up-regulated protein was Kir4.1 (gene name: kcnj10) (Fig. 1B), an inward rectifying potassium channel found on astrocytes and majorly responsible for setting their resting membrane potentials (RMPs) (Djukic et al. 2007; Olsen and Sontheimer 2008; Chever et al. 2010; Hibino et al. 2010; Sibille et al. 2015). Furthermore, it is proposed to buffer extracellular potassium in the tripartite synapses, where astrocytes wrap around synaptic sites (Halassa et al. 2007; Bedner and Steinhäuser 2013; Navarrete and Araque 2014; Papouin et al. 2017).

We then confirmed by western blot analysis and electrophysiology that the expression and conductance of Kir4.1 were indeed enhanced in two well-accepted animal models of depression: that is, cLH rats (Fig. 1B-E) and lipopolysaccharide (LPS)-induced depressive-like Wistar rats (Cui et al. 2018).

\section{BIDIRECTIONAL MODULATION OF DEPRESSION-LIKE BEHAVIORS BY GAIN AND LOSS OF FUNCTION OF ASTROCYTIC Kir4.1 IN THE LHb}

To test the consequences of the specific up-regulation of Kir4.1 in the LHb astrocytes, we used a strategy based on adeno-associated viruses $2 / 5$ (AAV2/5) and gfaABC1D, an astrocyte-specific human glial fibrillary acidic protein (GFAP) promoter (Fig. 2A). Viral infection of AAV-
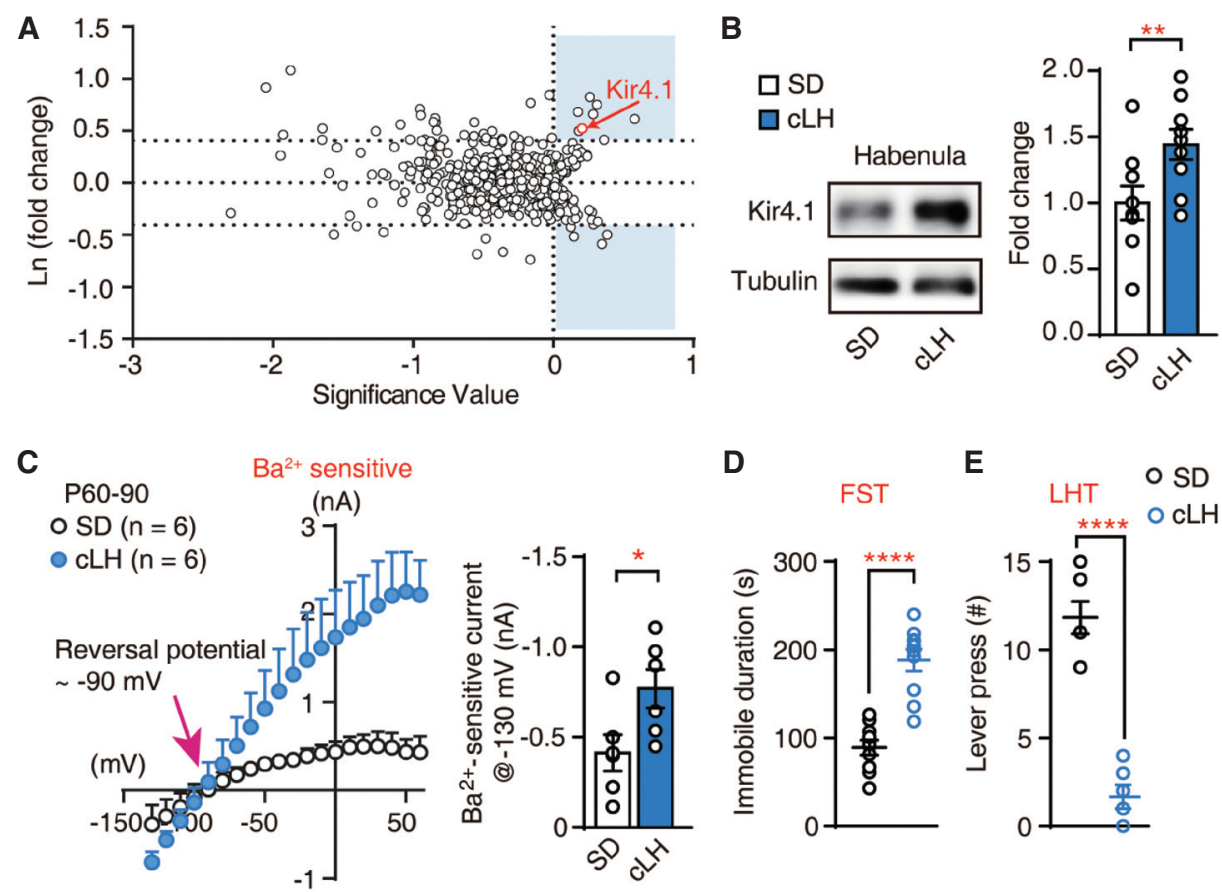

Figure 1. Up-regulation of Kir4.1 in the LHb in rat models of depression. $(A)$ Volcano plot of high-throughput proteomic screen identifying proteins that are differentially expressed in the habenulae of cLH rats versus wild-type rats. Ln (fold change) is the lntransformed value of the normalized protein ratio of cLH and control. Significance value was calculated as the average normalized ratio minus twofold SD. Proteins in the shaded areas exhibited $>50 \%$ significant change. Kir4.1 was one of the eight up-regulated proteins identified. Horizontal dashed lines indicate fold change of 50\%. (B) Western blot analysis showing up-regulation of the Kir4.1 protein in the membrane fraction of the habenula of cLH rats. $(C) \mathrm{I}-\mathrm{V}$ plot and bar graph showing $\mathrm{Ba}^{2+}$-sensitive Kir currents in the astrocytes of cLH and wild-type control rats at the age of P60-90. $(D, E)$ Forced swim test (FST) $(D)$ and learned helplessness test (LHT) $(E)$ phenotypes of cLH rats. 
A

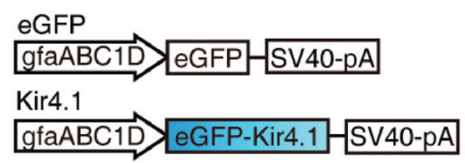

Ctrl-shRNA

Hi) Luciferase-shRNA CAG eGFP pA

Kir4.1-shRNA

Hi Kir4.1-shRNA $\overrightarrow{C A G}$ eGFP pA

eGFP

afaABC1D $>$ eGFP $p$

dnKir4.1

gfaABC1D dnKir4.1-2A-eGFP pA

127 TTIGYGF 133

to AAA

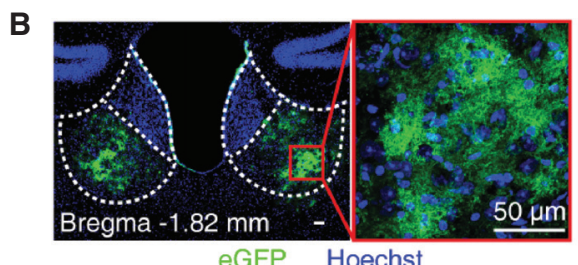

eGFP Hoechst

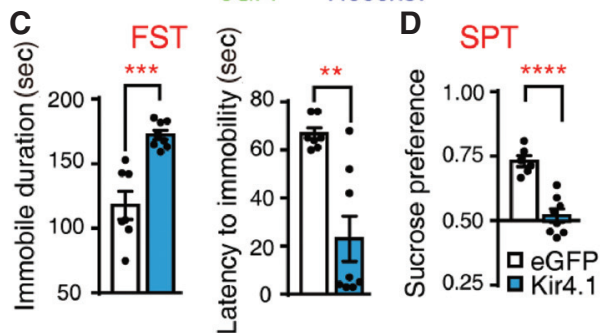

E $\quad$ FST $\quad F \quad$ LHT

口Ctrl-shRNA $\square$ Kir4.1-shRNA DeGFP $\square$ dnKir4.1

G -\#press $<5(\mathrm{LH}) \quad$ H

口\# press $5 \sim 10$

口 \# press $>10(\mathrm{NLH})$
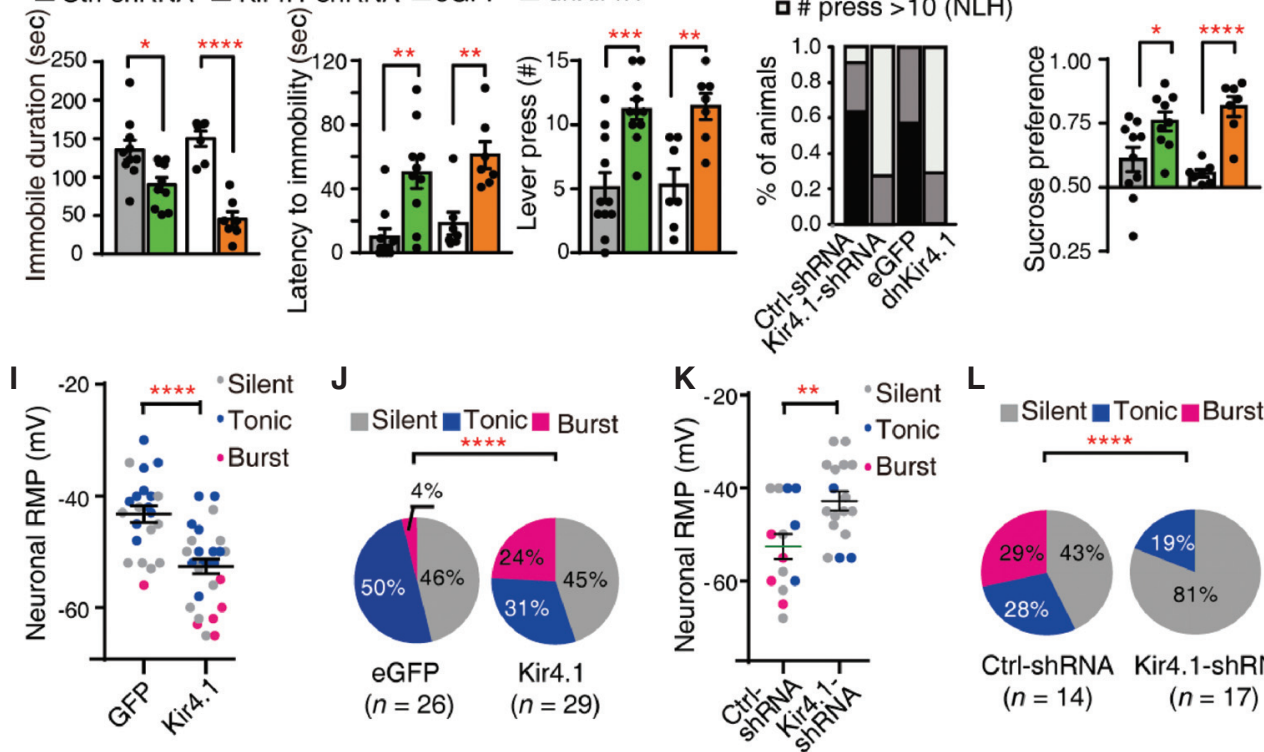

$\mathbf{L}$

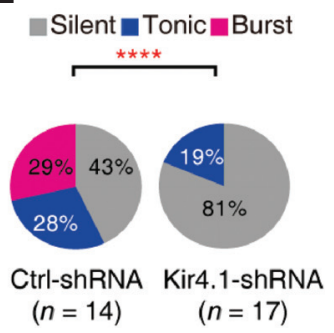

Figure 2. Bidirectional modulation of depression-like behaviors by gain and loss of function of astrocytic Kir4.1 in the LHb. $(A)$ Schematics of AAV vectors engineered to overexpress Kir4.1, Kir4.1 shRNA, or dominant-negative Kir4.1 and their corresponding controls. $(B)$ Bilateral viral injection of AAV-GFAP::Kir4.1 in mouse LHb (stained with antibody against GFP and Hoechst). (C,D) Behavioral effects of expression of Kir4.1-viral construct in the LHb in the FST $(C)$ and SPT $(D)$. $(E-H)$ Behavioral effects of expression of Kir4.1 loss-of-function-viral constructs in the LHb of cLH rats in the FST $(E)$, LHT $(F, G)$, and SPT $(H)$. $(G)$ Percentage of rats in each category. (LH) Learned helpless rats with fewer than five lever presses; (NLH) non-learned helpless rats with more than 10 lever presses. $(I, J)$ Scatter plots and pie charts showing that overexpression of Kir4.1 in LHb astrocytes leads to hyperpolarization of RMP $(I)$ and enhanced bursting in LHb neurons $(J)$. $(K, L)$ AAV-Kir4.1-shRNA viral infection in LHb astrocytes depolarizes neurons $(K)$ and abolishes neuronal bursting $(L)$.

GFAP::Kir4.1 led to its overexpression in astrocytes throughout the LHb (Fig. 2B). Infected mice displayed severe depression-like behaviors, including increased immobility in the forced swim test (FST; Fig. 2C) and decreased sucrose preference in the sucrose preference test (SPT; Fig. 2D), which model behavioral despair and anhedonia aspects of depression, respectively.

To test whether loss of function of Kir4.1 in the LHb could reverse depressive-like phenotypes, we devised two strategies, expressing either a short hairpin RNA (shRNA) to knock down Kir4.1 (AAV-H1::Kir4.1-shRNA) or a dominant-negative form of Kir4.1 (AAV-GFAP::dnKir4.1) to block its function (Fig. 2A). Both strategies caused a pronounced reduction in the depression-like phenotypes of cLH rats in three depression paradigms, including FST, learned helplessness (LH), and SPT (Fig. 2E-H).

\section{INCREASED LHb BURSTING ACTIVITY UNDERLYING ANIMAL MODELS OF DEPRESSION}

Consistent with the robust behavioral effects, at the cellular level, Kir4.1 gain and loss of function also caused strong phenotypes. When specifically overexpress- 
ing Kir4.1 just in the astrocytes of the LHb, we observed decreased RMP and increased burst firing in the LHb neurons (Fig. 2I,J). Vice versa, loss of Kir4.1 function depolarized neurons and eliminated bursting (Fig. 2K,L).

To investigate the potential link between increased LHb bursting activity and depression, we recorded spontaneous neural activity in LHb brain slices in several animal models of depression, including cLH rats and mice after chronic restraint stress (CRS). As shown previously, LHb neurons are intrinsically active and fall into three categories: silent, tonic firing, and burst firing (Fig. 3A-C; Weiss and Veh 2011). We found that in the LHb of cLH rats and CRS mice, the percentage of bursting neurons is significantly increased, by more than $100 \%$, compared with that in the control animals (Fig. 3D,E). Similar increased burst firing and enhanced network synchronization were also observed with in vivo recordings in CRS mice (Yang et al. 2018a).

To investigate whether the increased LHb burst firing was sufficient to induce depressive-like behaviors, we used optogenetics to drive LHb bursts (Fig. 3F). Based on the rebound mechanism (see below) of LHb bursts, we used an inhibitory opsin (eNpHR3.0, an enhanced variant of halorhodopsin) and short pulsed yellow light $(1 \mathrm{~Hz}$, $100 \mathrm{msec}$ ) to transiently hyperpolarize LHb neurons. At each termination of yellow light, as the membrane potential rebounded, bursts were induced with a high success rate (Fig. 3G,H). Behaviorally, this optogenetic protocol induced both real-time place aversion (RTPA) (Fig. 3I,L) and depressive-like behaviors in the FST (Fig. 3J,M) and SPT (Fig. 3K,N).

\section{LHb BURSTS AS NOVEL TARGETS FOR RAPID ANTIDEPRESSANT KETAMINE}

To test the effects of rapid antidepressant ketamine on LHb bursts, we administered systemic injections of ketamine before animal sacrifice for brain slice recordings. We found ketamine reversed bursting to the control level in both cLH rats and CRS mice (Fig. 3D,E). These results provide the first hint that ketamine may act on LHb bursts.

A second hint came from a challenging LHb cannular experiment, in which we infused drugs into the $\mathrm{LHb}$ and tested the effects on depressive-like behaviors. For the positive control, we reasoned that as the $\mathrm{LHb}$ is hyperactive in depression, blocking most of its excitatory inputs with the $\alpha$-amino-3-hydroxy-5-methyl-4-isoxazole-propionic acid receptor (AMPAR) blocker NBQX (1,2,3,4-tetrahydro-6-nitro-2,3-dioxo-benzo[f] quinoxaline-7-sulfonamide) should theoretically cause antidepressant effects. Out of curiosity, we tested the NMDAR blocker, AP5 (2amino-5-phosphonopentanoic acid), in a parallel experiment. Surprisingly, AP5 but not NBQX produced a strong antidepressant effect in the FST when locally infused into the LHb (Fig. 4A-D). Knowing that ketamine is also a blocker of NMDAR, we then tested the behavioral effects caused by local infusion of ketamine into the LHb. In both the FST and SPT, ketamine rapidly alleviated depressivelike symptoms within $1 \mathrm{~h}$ of local bilateral infusion into the
LHb of cLH rats (Fig. 4E,F). This was achieved at a behaviorally relevant dose $(\sim 5 \mu \mathrm{M})$, as shown by liquid chromatography-tandem mass spectrometry (Fig. 4G). To the best of our knowledge, this is the first evidence that ketamine can cause rapid antidepressant effects within this short interval from within just one brain area.

Given that NMDAR-mediated calcium influx plays a pivotal role in burst generation in several brain regions (Grillner et al. 1981; Schiller et al. 2000; Zhu et al. 2005), and considering the above two findings, we next tested whether NMDARs were directly required for the $\mathrm{LHb}$ bursting activity. Bath application of ketamine (100 $\mu \mathrm{M})$, as well as the more specific NMDAR antagonist AP5, completely eliminated spontaneous burst firing within seconds of application (Fig. 5A,B). At more behaviorally relevant concentrations $(\sim 1-10 \mu \mathrm{M})$, ketamine still blocked LHb bursts but takes minutes to cause complete blockade (Yang et al. 2018a). The classical selective serotonin reuptake inhibitor (SSRI)-type antidepressant, fluoxetine, does not instantly block LHb bursts (Yang et al. 2018a) but reduces bursts after chronic treatment (data unpublished), suggesting that reduced burst firing of LHb neurons may be a common end point for antidepressant drugs to exert their efficacy.

Interestingly, consistent with the modest behavioral effects of cannular infusion (Fig. 4C,D), full blockade of AMPARs with NBQX $(10 \mu \mathrm{M})$ only reduced bursts moderately (by $20 \%$; Fig. 5C). In addition, the application of AMPA or blockade of GABA receptors (GABARs) also increased LHb burst firing by a similar extent (Yang et al. 2018a). These results indicate that LHb bursting was mostly driven by the intrinsic properties of the neuron and only moderately modified by synaptic inputs from AMPARs or GABARs. This is consistent with previous research showing multiple mechanisms for the generation of burst firing (McCormick and Huguenard 1992; Kepecs and Lisman 2003; Krahe and Gabbiani 2004; Peña et al. 2004; Major et al. 2013).

\section{IONOIC MECHANISM OF LHb BURSTS}

In addition to NMDARs, we also found that LHb bursts were dependent on a hyperpolarized membrane potential and the T-VSCC. The membrane potential of LHb bursting neurons is more hyperpolarized than that of the silent or tonic-firing types (Wilcox et al. 1988; Weiss and Veh 2011; Yang et al. 2018a). A hyperpolarizing or depolarizing current injection can convert originally tonic-firing neurons into bursting mode or vice versa, respectively (Yang et al. 2018a).

The average RMP of bursting neurons was $61.3 \pm 1.9 \mathrm{mV}$ in $\mathrm{SD}$ and $\mathrm{cLH}$ rats, within a range that can de-inactivate the T-VSCCs. One prominent feature of the pacemaker channel T-VSCC is that it inactivates quickly after opening but can be de-inactivated to initiate burst firing when the membrane potential is hyperpolarized below $-55 \mathrm{mV}$ for $>100$ msec (McCormick and Huguenard 1992). Indeed, bath application of the specific T-VSCC blocker mibefradil $(10 \mu \mathrm{M})$ onto the $\mathrm{LHb}$ brain slices effectively decreased 
A

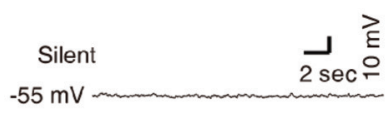

B
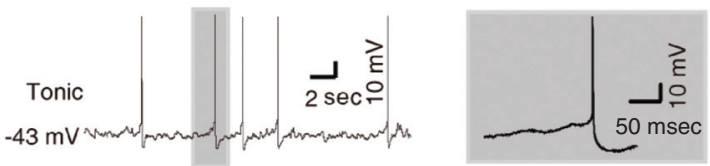

C

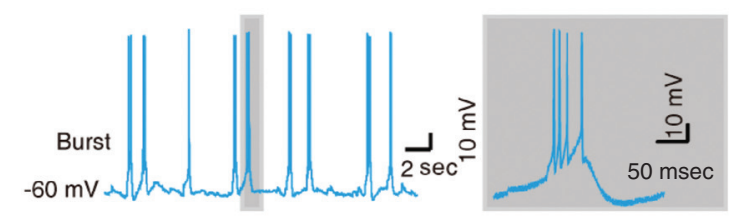

D Silent $_{* *} \square$ Tonic $_{*}$ Burst

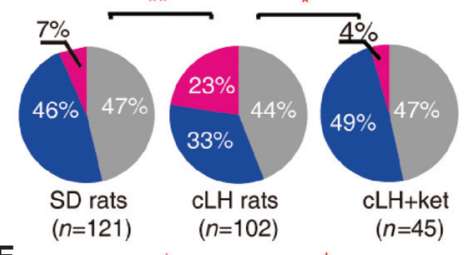

E

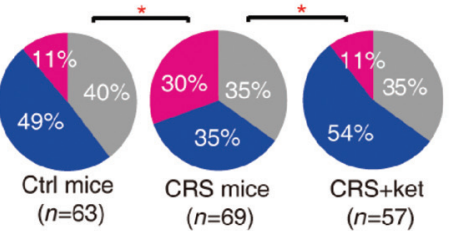

H $1 \mathrm{~Hz}, 100 \mathrm{msec}, 589 \mathrm{~nm}$
$\mathbf{F}$

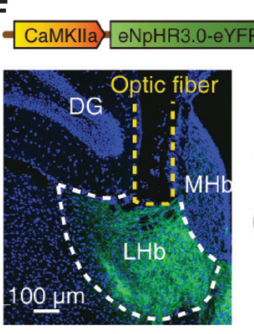

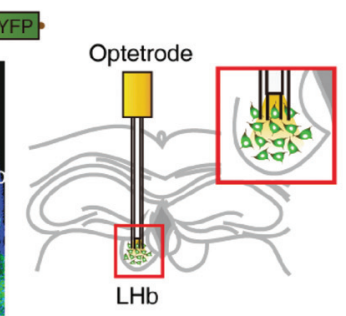
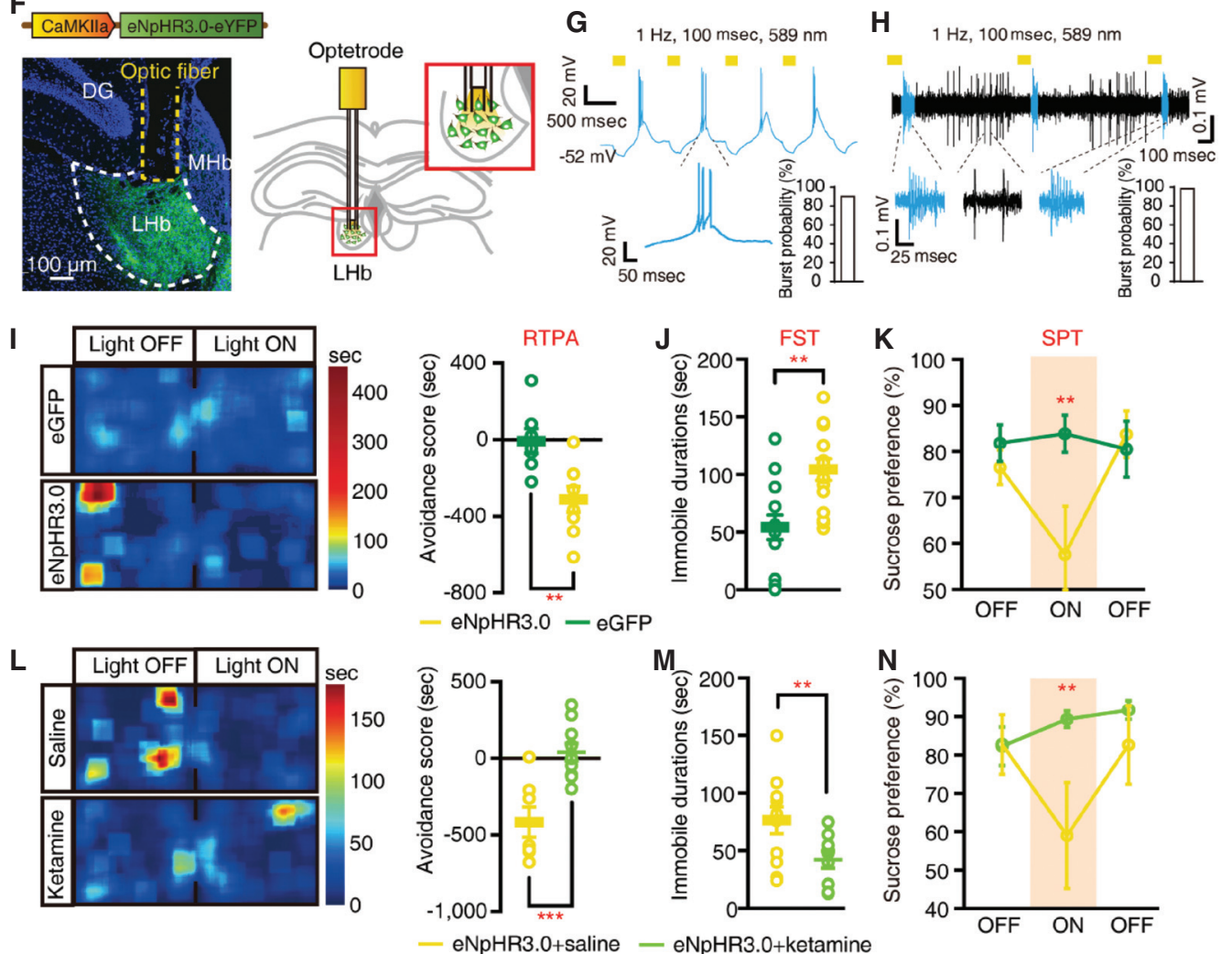

Figure 3. Enhanced LHb bursting in animal models of depression is sufficient to drive aversion and depression-like behaviors. $(A-C)$ Representative traces showing spontaneous activity of silent $(A)$, tonic-firing $(B)$, and burst-firing $(C)$ LHb neurons recorded with whole-cell patch clamps. $(D, E)$ Pie charts illustrating the percentage abundance of the three types of LHb neurons. $(F-N)$ eNpHR3.0-induced rebound bursting drives behavioral aversion and depressive-like symptoms that are reversible by ketamine. $(F)$ Construct of AAV2/9-eNpHR3.0 (left top), example site of viral injection and optic fiber implantation (left bottom), and illustration of optetrode recording $(r i g h t)$. $(G, H)$ Representative traces showing rebound bursts reliably elicited by pulsed yellow light in LHb brain slices in vitro $(G)$ and in vivo $(H)$ from mice infected with AAV2/9-eNpHR3.0. Spikes in bursting and tonic-firing mode are shown in blue and black, respectively. Percentage of successfully induced bursts is shown at bottom right. (I,L) RTPA induced by eNpHR3.0driven bursts $(I)$ reversed by i.p. injection of ketamine (1 h prior; $L$ ). (Left) Representative heat maps of RTPA; (right) quantitative aversion score. $(J, K, M, N)$ Depression-like behaviors in the FST $(J)$ and SPT $(K)$ induced by eNpHR3.0-driven bursts reversed by i.p. injection of ketamine ( $1 \mathrm{~h}$ prior; $M, N)$.

the bursting probability and reduced the amplitude of the plateau potentials of spontaneous bursts (Fig. 5D), without significantly changing the RMPs (Yang et al. 2018a).

To understand how NMDARs and T-VSCCs work synergistically to mediate LHb burst firing, we constructed a minimal biophysical model that successfully recapitulated the key characteristics of LHb bursts, including the voltage dependency and in silico knockout effects of T-VSCCs and NMDARs (Yang et al. 2018a). Briefly, activation of T-VSCCs removes the $\mathrm{Mg}^{2+}$ blockade of NMDARs, and the opening of these two channels synergistically drives membrane potential toward the threshold for a burst of 
A

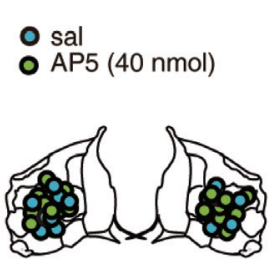

E
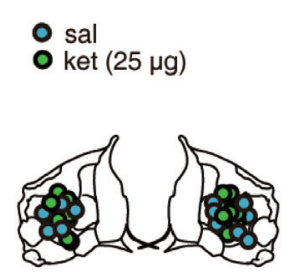
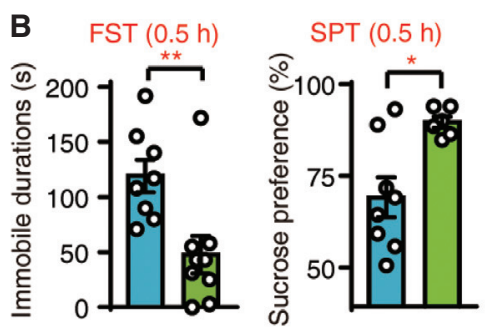

C

o sal

- NBQX (1 nmol)
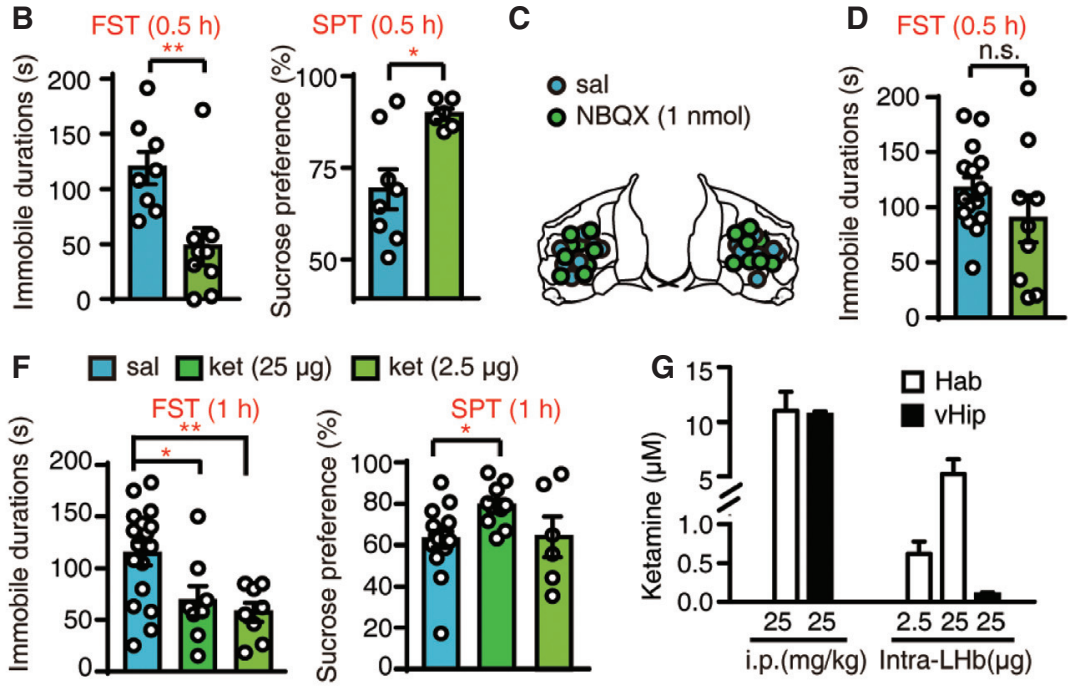
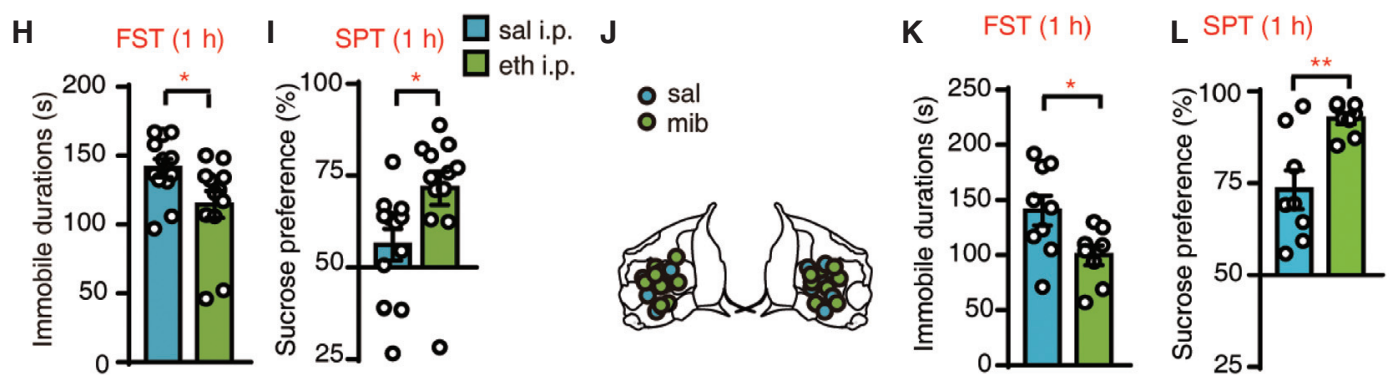

Figure 4. Local infusion of ketamine and mibefradil into LHb causes rapid antidepressant effects. $(A-F)$ Acute effects of local bilateral $\mathrm{LHb}$ infusion of $\mathrm{AP} 5(A, B), \mathrm{NBQX}(C, D)$, or ketamine $(E, F)$ in FST and SPT. Infusion sites of drugs verified by cholera toxin subunit $\mathrm{B}$ $(\mathrm{CTB})(A, C, E) .(G)$ Concentration of ketamine in habenula (Hab) or ventral hippocampus (vHip) $1 \mathrm{~h}$ after i.p. injection or intra-LHb infusion of ketamine, as measured by LC-MS/MS. (H-L) Antidepressant effects caused by systemic ethosuximide injection (200 mg $\mathrm{kg}^{-1}$, i.p.; $\left.H, I\right)$ or local bilateral infusion of mibefradil into the LHb $(J-L)$ in the FST $(H, K)$ and SPT $(I, L) 1 \mathrm{~h}$ after treatment. Infusion sites verified by CTB $(J)$.

APs. As the RMP falls below $-55 \mathrm{mV}$, it de-inactivates the $\mathrm{T}$-VSCCs, resulting in the intrinsic propensity of the LHb neurons to initiate another burst cycle (Fig. 5E).

One strong prediction from the above results is that blockade of T-VSCC may also be rapidly antidepressant. Indeed, both systematic injection of the T-VSCC blocker 2-ethyl-2-methylsuccinimide (ethosuximide), which can cross the blood-brain barrier, in CRS mice and bilateral infusion of mibefradil $(10 \mathrm{nmol})$ into the $\mathrm{LHb}$ of cLH rats both caused rapid antidepressant effects in the FST and SPT (Fig. 4H-L).

\section{Kir4.1 BUFFERS PERISOMATIC POTASSIUM TO REGULATE RMP AND BURSTING ACTIVITY OF LHb NEURONS}

The final issue is how an astrocytic $\mathrm{K}$ channel regulates the RMP and burst firing of LHb neurons to cause depression. Several previous studies have indicated that astrocytes may play a key role in neuronal rhythmic bursting activity in the central pattern generator: for example, astrocytes have been found to regulate bursting activity by adjusting extracellular calcium concentration (Okada et al. 2012; Morquette et al. 2015) or by controlling pH- dependent release of ATP (Gourine et al. 2010). Here we found a new form of glia-neuron interaction in setting the neuronal firing mode in the $\mathrm{LHb}$ under a depression-like state.

When we examined the expression pattern of Kir4.1, we found that unlike in the hippocampus, the Kir4.1 immunohistochemical signals in the LHb showed a unique pattern, wrapping around the NeuN signals at neuronal cell bodies (Fig. 6A). Electron microscopy imaging further confirmed that Kir4.1-positive gold particles encircled the membrane of neuronal soma (Fig. 6B). We propose that this perisomatic expression and the highly confined extracellular space in the $\mathrm{LHb}$ ensure that Kir4.1 can efficiently buffer the potassium released from bursting neurons. Specifically, we predicted that reducing Kir4.1 function should lead to increased extracellular potassium $\left(\mathrm{K}_{\text {[out] }}\right)$ and, according to the Nernst equation, more depolarized RMP. Indeed, blocking Kir4.1 with $\mathrm{Ba}^{2+}$ increased the RMPs and stopped neurons from burst firing (Cui et al. 2018). Furthermore, by increasing astrocytic Kir4.1 expression or decreasing $\mathrm{K}_{\text {[out] }}$, we were able to phenocopy in wild-type animals several key neuronal properties observed in the LHb of animal models of depression - namely, hyperpolarized RMPs and enhanced bursts. These results indicate 
A

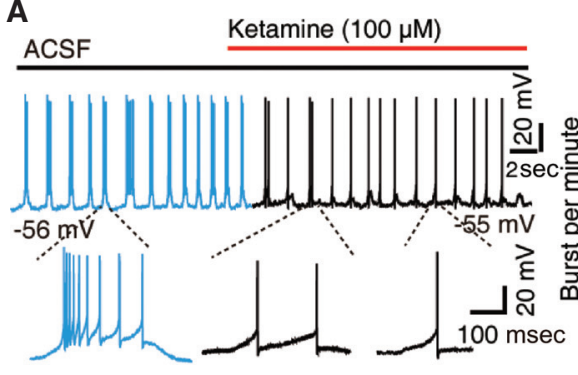

C

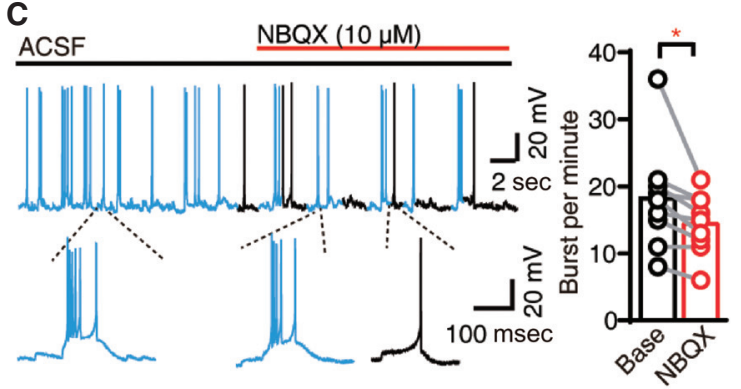

B

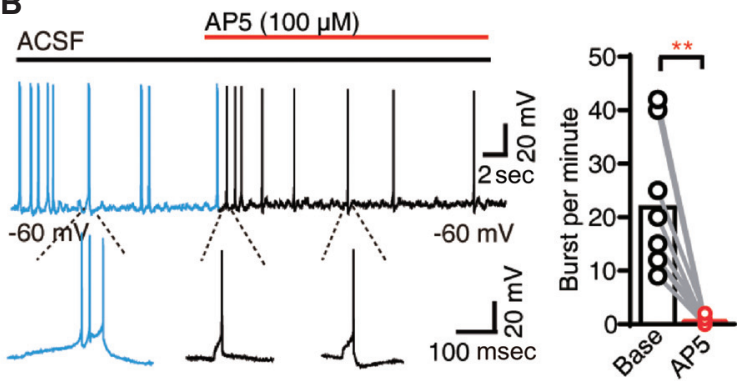

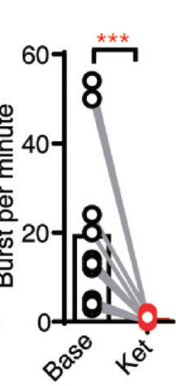

D
Mibefradil $(10 \mu \mathrm{M})$

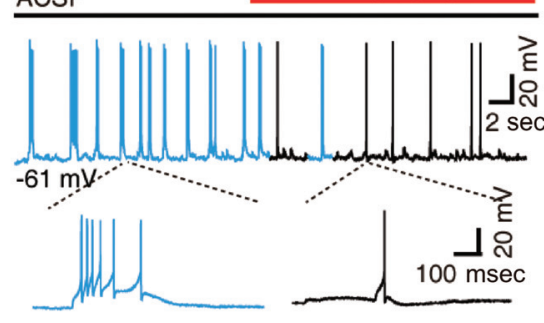

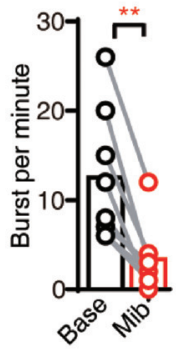

E

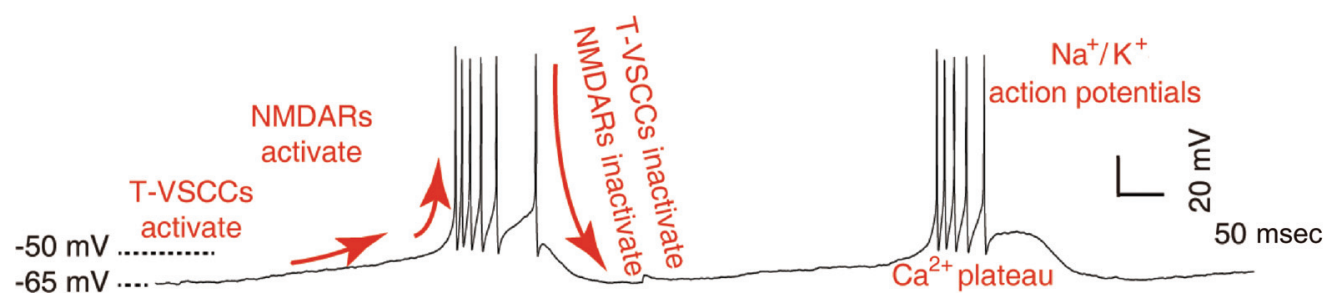

Figure 5. LHb bursts rely on synergistic activation of NMDAR and T-VSCC. $(A-D)$ Example traces $($ left) and statistics (right) (sampled within 3 min before and $1 \mathrm{~min}$ after drug application) showing effects of ketamine $(A)$, AP5 $(B)$, NBQX $(C)$, and mibefradil $(D)$ on spontaneous bursts in the LHb of wild-type and cLH rats. Spikes in bursting and tonic-firing mode are shown in blue and black, respectively. $(E)$ Example trace summarizing the hypothetical ionic components and channel mechanisms involved in LHb bursting: hyperpolarization of neurons to membrane potentials negative to $-55 \mathrm{mV}$ de-inactivates T-VSCC. Calcium current continues to grow as the de-inactivated T-VSCCs increase, leading to a transient $\mathrm{Ca}^{2+}$ plateau potential. The $\mathrm{Ca}^{2+}$ plateau helps remove the magnesium blockade of NMDARs, whereas T-VSCC inactivates rapidly during the depolarization. After the $\mathrm{Ca}^{2+}$ plateau reaches approximately $-45 \mathrm{mV}$, NMDA current dominates the driving force to further depolarize RMP to the threshold for $\mathrm{Na}^{+}$spike generation. As RMP falls back to below $-55 \mathrm{mV}$, it de-inactivates T-VSCC, priming LHb neurons to generate the next cycle of bursting.

A

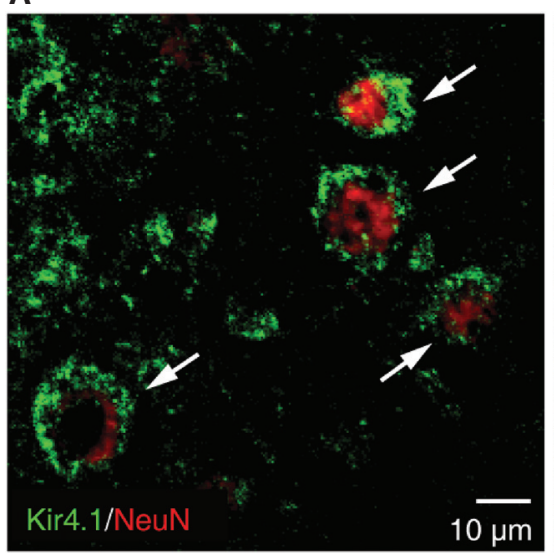

B

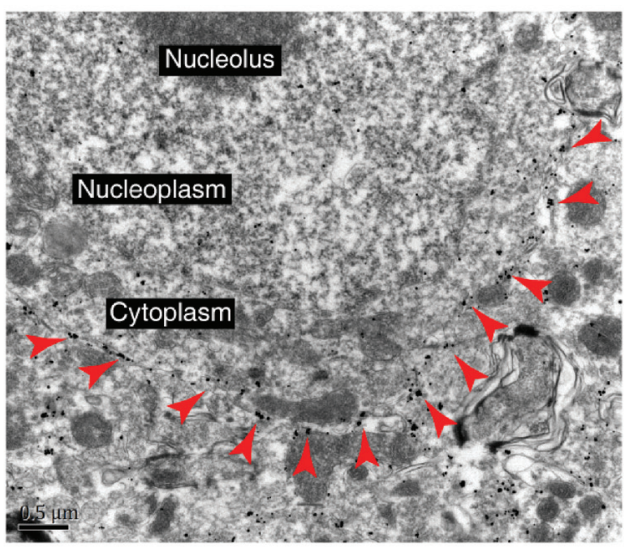

Figure 6. Kir4.1 is expressed on astrocytic processes enveloping neuronal soma in the LHb. $(A)$ Kir4.1 envelope neuronal somata as indicated by white arrows (three independent experiments). (B) Immunogold electron microscopy of Kir4.1. Red arrows indicate gold signals surrounding a neuronal soma (three independent experiments). 


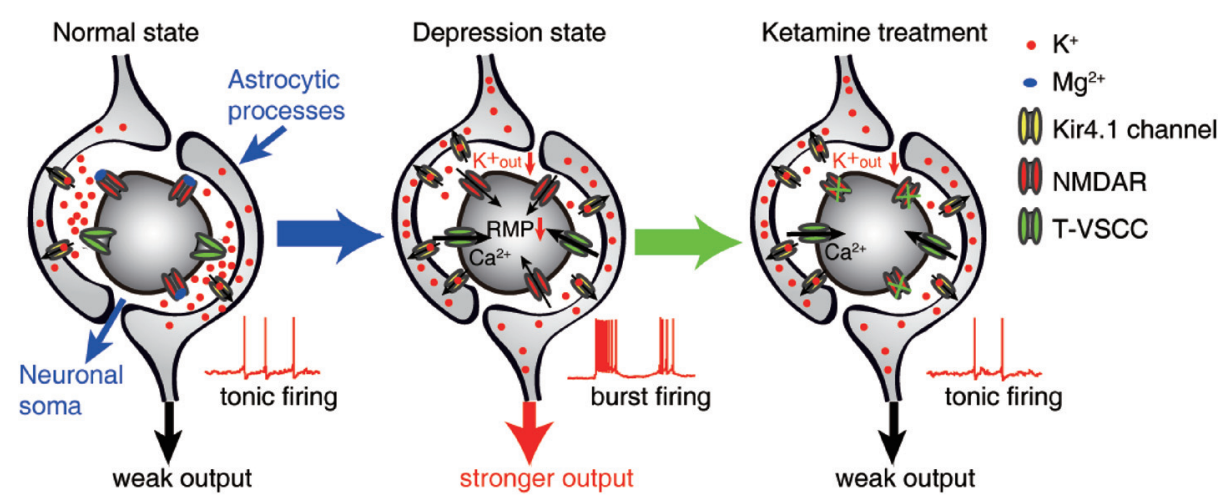

Figure 7. Model for mechanisms of depression and ketamine treatment in the LHb. Up-regulation of Kir4.1 on astrocytic processes surrounding neuronal somata leads to enhanced $\mathrm{K}^{+}$buffering at the tight neuron-glia junction, decreased $\mathrm{K}_{\text {[out] }}$, and hyperpolarized neuronal RMP. Consequently, de-inactivation of T-VSCCs initiates NMDAR-dependent bursts, causing a stronger output of the LHb to trigger depression. Ketamine blockade of NMDARs stops bursting and relieves depression. We hypothesize that for highly active neurons, like in the LHb, an efficient potassium buffering system is needed at the neuron-glia interface to clear out the constantly released potassium. After prolonged emotional stress, the up-regulation of Kir4.1 leads to lowered extracellular potassium and hyperpolarized neuronal RMP. Consequently, de-inactivation of T-VSCCs initiates NMDAR-dependent bursts. As bursts can reduce synaptic failure and synchronize network output, they will generate stronger output to inhibit downstream aminergic centers. Finally, blockade of NMDARs by ketamine eliminates LHb burst firing, thereby releasing the break onto the reward centers to improve mood.

that enhanced extracellular $\mathrm{K}^{+}$clearance resulting from the up-regulation of Kir4.1 might underlie the neuronal hyperpolarization required for burst initiation in the $\mathrm{LHb}$.

Much previous work on neuron-glia interaction and Kir4.1 function has focused on the tripartite synapses, where astrocytes wrap around synaptic sites (Halassa et al. 2007; Bedner and Steinhäuser 2013; Navarrete and Araque 2014; Papouin et al. 2017). Here we identified a new mechanism operating at neuronal somata, which powerfully regulates neuronal excitability.

\section{CONCLUDING REMARKS}

By combining an unbiased, high-throughput, quantitative proteomic screen with pharmacology, in vitro and in vivo electrophysiology recording, viral manipulation, and behavioral analysis, we identified a critical function of the glia-specific ion channel, Kir4.1, operating in the highly specialized perisomatic extracellular space in the LHb, as a powerful molecular determinant of depression. We revealed a novel form of glia-neuron interaction in setting the neuronal firing pattern, in particular the bursting pattern, which is crucial in driving depressive-like behaviors. Our results also suggest a simple and plausible model in which ketamine exerts its rapid antidepressant effects by blocking bursting activity of $\mathrm{LHb}$ neurons to disinhibit downstream monoaminergic centers (Fig. 7). In addition, these results revealed T-VSCCs and Kir4.1 as promising targets for the development of new rapid-acting antidepressants.

\section{ACKNOWLEDGMENTS}

This study was supported by grants from the National Natural Science Foundation of China (31830032, 81527901, 31225010, and 91432108 to H.H. and 81701335 to Y.C.), the Non-profit Central Research Institute Fund of Chinese Academy of Medical Sciences
(2017PT31038, 2018PT31041), and the National Key R\&D Program of China (2016YFA0501000 to H.H).

\section{REFERENCES}

Banasr M, Dwyer JM, Duman RS. 2011. Cell atrophy and loss in depression: reversal by antidepressant treatment. Curr Opin Cell Biol 23: 730-737. doi:10.1016/j.ceb.2011.09.002

Bedner P, Steinhäuser C. 2013. Altered Kir and gap junction channels in temporal lobe epilepsy. Neurochem Int 63: 682687. doi:10.1016/j.neuint.2013.01.011

Belykh I, de Lange E, Hasler M. 2005. Synchronization of bursting neurons: what matters in the network topology. Phys Rev Lett 94: 188101. doi:10.1103/PhysRevLett.94.188101

Berman RM, Cappiello A, Anand A, Oren DA, Heninger GR, Charney DS, Krystal JH. 2000. Antidepressant effects of ketamine in depressed patients. Biol Psychiat 47: 351-354.

Bromberg-Martin ES, Hikosaka O. 2009. Midbrain dopamine neurons signal preference for advance information about upcoming rewards. Neuron 63: 119-126. doi:10.1016/j.neuron .2009 .06 .009

Caldecott-Hazard S, Mazziotta J, Phelps M. 1988. Cerebral correlates of depressed behavior in rats, visualized using ${ }^{14} \mathrm{C}-2-$ deoxyglucose autoradiography. J Neurosci 8: 1951-1961. doi:10.1523/JNEUROSCI.08-06-01951.1988

Chever O, Djukic B, McCarthy KD, Amzica F. 2010. Implication of Kir4.1 channel in excess potassium clearance: an in vivo study on anesthetized glial-conditional Kir4.1 knock-out mice. J Neurosci 30: 15769-15777. doi:10.1523/JNEURO SCI.2078-10.2010

Cui YH, Yang Y, Ni ZY, Dong YY, Cai GH, Foncelle A, Ma SS, Sang KN, Tang SY, Li YZ, et al. 2018. Astroglial Kir4.1 in the lateral habenula drives neuronal bursts in depression. Nature 554: 323-327. doi:10.1038/nature 25752

Cui YH, Hu SH, Hu HL. 2019. Lateral habenular burst firing as a target of the rapid antidepressant effects of ketamine. Trends Neurosci, in press.

DiazGranados N, Ibrahim L, Brutsche NE, Newberg A, Kronstein P, Khalife S, Kammerer WA, Quezado Z, Luckenbaugh DA, Salvadore G, et al. 2010a. A randomized add-on trial of an $N$-methyl-D-aspartate antagonist in treatment-resistant bipolar depression. Arch Gen Psychiat 67: 793-802.

DiazGranados N, Ibrahim LA, Brutsche NE, Ameli R, Henter ID, Luckenbaugh DA, Machado-Vieira R, Zarate CA. 2010 b. 
Rapid resolution of suicidal ideation after a single infusion of an $N$-methyl-D-aspartate antagonist in patients with treatmentresistant major depressive disorder. J Clin Psychiat 71: 16051611.

Djukic B, Casper KB, Philpot BD, Chin LS, McCarthy KD. 2007. Conditional knock-out of Kir4.1 leads to glial membrane depolarization, inhibition of potassium and glutamate uptake, and enhanced short-term synaptic potentiation. $J$ Neurosci 27: 11354-11365. doi:10.1523/JNEUROSCI.072307.2007

Duman RS, Li N. 2012. A neurotrophic hypothesis of depression: role of synaptogenesis in the actions of NMDA receptor antagonists. Philos Trans R Soc Lond B Biol Sci 367: 24752484. doi:10.1098/rstb.2011.0357

Gourine AV, Kasymov V, Marina N, Tang F, Figueiredo MF, Lane S, Teschemacher AG, Spyer KM, Deisseroth K, Kasparov S. 2010. Astrocytes control breathing through $\mathrm{pH}$-dependent release of ATP. Science 329: 571-575. doi:10.1126/science. 1190721

Grillner S, McClellan A, Sigvardt K, Wallén P, Wilén M. 1981. Activation of NMDA-receptors elicits "fictive locomotion" in lamprey spinal cord in vitro. Acta Physiol Scand 113: 549551 .

Halassa MM, Fellin T, Haydon PG. 2007. The tripartite synapse: roles for gliotransmission in health and disease. Trends Mol Med 13: 54-63. doi:10.1016/j.molmed.2006.12.005

Harris KD, Hirase H, Leinekugel X, Henze DA, Buzsáki G. 2001. Temporal interaction between single spikes and complex spike bursts in hippocampal pyramidal cells. Neuron 32: 141149. doi:10.1016/S0896-6273(01)00447-0

Henn FA, Vollmayr B. 2005. Stress models of depression: forming genetically vulnerable strains. Neurosci Biobehav Rev 29: 799-804. doi:10.1016/j.neubiorev.2005.03.019

Hibino H, Inanobe A, Furutani K, Murakami S, Findlay I, Kurachi Y. 2010. Inwardly rectifying potassium channels: their structure, function, and physiological roles. Physiol Rev 90: 291-366. doi:10.1152/physrev.00021.2009

Hikosaka O. 2010. The habenula: from stress evasion to valuebased decision-making. Nat Rev Neurosci 11: 503-513. doi: $10.1038 / \mathrm{nrn} 2866$

Hikosaka O, Sesack SR, Lecourtier L, Shepard PD. 2008 Habenula: crossroad between the basal ganglia and the limbic system. J Neurosci 28: 11825-11829. doi:10.1523/JNEURO SCI.3463-08.2008

Holtzheimer PE, Mayberg HS. 2011. Deep brain stimulation for psychiatric disorders. Annu Rev Neurosci 34: 289-307. doi: 10.1146/annurev-neuro-061010-113638

Hu H. 2016. Reward and aversion. Annu Rev Neurosci 39: 297 324. doi:10.1146/annurev-neuro-070815-014106

Huang L, Xi Y, Peng YF, Yang Y, Huang XD, Fu YW, Tao Q, Xiao J, Yuan TF, An K, et al. 2019. A visual circuit related to habenula underlies antidepressive effects of light therapy. Neuron, in press. doi:10.1016/j.neuron.2019.01.037

Izhikevich EM, Desai NS, Walcott EC, Hoppensteadt FC. 2003. Bursts as a unit of neural information: selective communication via resonance. Trends Neurosci 26: 161-167. doi: 10.1016/S0166-2236(03)00034-1

Kepecs A, Lisman J. 2003. Information encoding and computation with spikes and bursts. Network 14: 103-118. doi: 10.1080/net.14.1.103.118

Krahe R, Gabbiani F. 2004. Burst firing in sensory systems. Nat Rev Neurosci 5: 13-23. doi:10.1038/nrn1296

Lammel S, Lim BK, Ran C, Huang KW, Betley MJ, Tye KM, Deisseroth K, Malenka RC. 2012. Input-specific control of reward and aversion in the ventral tegmental area. Nature 491: 212-217. doi:10.1038/nature11527

Lecca S, Pelosi A, Tchenio A, Moutkine I, Lujan R, Hervé D, Mameli M. 2016. Rescue of GABAB and GIRK function in the lateral habenula by protein phosphatase $2 \mathrm{~A}$ inhibition ameliorates depression-like phenotypes in mice. Nat Med 22: 254 261. doi: $10.1038 / \mathrm{nm} .4037$

Li B, Piriz J, Mirrione M, Chung C, Proulx CD, Schulz D, Henn F, Malinow R. 2011. Synaptic potentiation onto habenula neu- rons in the learned helplessness model of depression. Nature 470: 535-539. doi:10.1038/nature09742

Li K, Zhou T, Liao L, Yang Z, Wong C, Henn F, Malinow R, Yates JR III, Hu H. 2013. $\beta$ CaMKII in lateral habenula mediates core symptoms of depression. Science 341: 1016-1020. doi:10.1126/science. 1240729

Lisman JE. 1997. Bursts as a unit of neural information: making unreliable synapses reliable. Trends Neurosci 20: 38-43. doi: 10.1016/S0166-2236(96)10070-9

Livingstone MS. 1996. Oscillatory firing and interneuronal correlations in squirrel monkey striate cortex. J Neurophysiol 75: 2467-2485. doi:10.1152/jn.1996.75.6.2467

Major G, Larkum ME, Schiller J. 2013. Active properties of neocortical pyramidal neuron dendrites. Annu Rev Neurosci 36: 1-24. doi:10.1146/annurev-neuro-062111-150343

Matsumoto M, Hikosaka O. 2007. Lateral habenula as a source of negative reward signals in dopamine neurons. Nature 447: 1111-1115. doi:10.1038/nature05860

McCormick DA, Huguenard JR. 1992. A model of the electrophysiological properties of thalamocortical relay neurons. $J$ Neurophysiol 68: 1384-1400. doi:10.1152/jn.1992.68.4. 1384

Morquette P, Verdier D, Kadala A, Féthière J, Philippe AG, Robitaille R, Kolta A. 2015. An astrocyte-dependent mechanism for neuronal rhythmogenesis. Nat Neurosci 18: 844-854. doi: $10.1038 / \mathrm{nn} .4013$

Morris JS, Smith KA, Cowen PJ, Friston KJ, Dolan RJ. 1999. Covariation of activity in habenula and dorsal raphé nuclei following tryptophan depletion. Neuroimage 10: 163-172. doi:10.1006/nimg.1999.0455

Navarrete M, Araque A. 2014. The Cajal school and the physiological role of astrocytes: a way of thinking. Front Neuroanat 8: 33. doi:10.3389/fnana.2014.00033

Nuno-Perez A, Tchenio A, Mameli M, Lecca S. 2018. Lateral habenula gone awry in depression: bridging cellular adaptations with therapeutics. Front Neurosci 12: 485. doi:10.3389/ fnins.2018.00485

Okada Y, Sasaki T, Oku Y, Takahashi N, Seki M, Ujita S, Tanaka KF, Matsuki N, Ikegaya Y. 2012. Preinspiratory calcium rise in putative pre-Botzinger complex astrocytes. J Physiol 590: 4933-4944. doi:10.1113/jphysiol.2012.231464

Olsen ML, Sontheimer H. 2008. Functional implications for Kir4.1 channels in glial biology: from $\mathrm{K}^{+}$buffering to cell differentiation. J Neurochem 107: 589-601. doi:10.1111/ j.1471-4159.2008.05615.x

Papouin T, Dunphy J, Tolman M, Foley JC, Haydon PG. 2017. Astrocytic control of synaptic function. Philos Trans $R$ Soc Lond B Biol Sci 372: 20160154. doi:10.1098/rstb.2016 .0154

Peña F, Parkis MA, Tryba AK, Ramirez JM. 2004. Differential contribution of pacemaker properties to the generation of respiratory rhythms during normoxia and hypoxia. Neuron 4: $105-117$.

Proulx CD, Hikosaka O, Malinow R. 2014. Reward processing by the lateral habenula in normal and depressive behaviors. Nat Neurosci 17: 1146-1152. doi:10.1038/nn.3779

Proulx CD, Aronson S, Milivojevic D, Molina C, Loi A, Monk B, Shabel SJ, Malinow R. 2018. A neural pathway controlling motivation to exert effort. Proc Natl Acad Sci 115: 5792-5797. doi:10.1073/pnas.1801837115

Ramirez JM, Tryba AK, Peña F. 2004. Pacemaker neurons and neuronal networks: an integrative view. Curr Opin Neurobiol 14: 665-674. doi:10.1016/j.conb.2004.10.011

Root DH, Mejias-Aponte CA, Zhang SL, Wang HL, Hoffman AF, Lupica CR, Morales M. 2014. Single rodent mesohabenular axons release glutamate and GABA. Nat Neurosci 17: 1543-1551. doi:10.1038/nn.3823

Russo SJ, Nestler EJ. 2013. The brain reward circuitry in mood disorders. Nat Rev Neurosci 14: 609-625. doi:10.1038/nrn 3381

Schiller J, Major G, Koester HJ, Schiller Y. 2000. NMDA spikes in basal dendrites of cortical pyramidal neurons. Nature 404: 285-289. 
Schultz W, Dayan P, Montague PR. 1997. A neural substrate of prediction and reward. Science 275: 1593-1599. doi:10.1126/ science.275.5306.1593

Seo JS, Zhong P, Liu A, Yan Z, Greengard P. 2018. Elevation of p11 in lateral habenula mediates depression-like behavior. $\mathrm{Mol}$ Psychiatry 23: 1113-1119. doi:10.1038/mp.2017.96

Shabel SJ, Proulx CD, Trias A, Murphy RT, Malinow R. 2012. Input to the lateral habenula from the basal ganglia is excitatory, aversive, and suppressed by serotonin. Neuron 74: 475481. doi:10.1016/j.neuron.2012.02.037

Sibille J, Dao Duc K, Holcman D, Rouach N. 2015. The neuroglial potassium cycle during neurotransmission: role of Kir4.1 channels. PLoS Comput Biol 11: e1004137. doi: 10.1371/journal.pcbi.1004137

Sorokin JM, Davidson TJ, Frechette E, Abramian AM, Deisseroth K, Huguenard JR, Paz JT. 2017. Bidirectional control of generalized epilepsy networks via rapid real-time switching of firing mode. Neuron 93: 194-210. doi:10.1016/j.neuron 2016.11.026

Stamatakis AM, Stuber GD. 2012. Activation of lateral habenula inputs to the ventral midbrain promotes behavioral avoidance. Nat Neurosci 15: 1105-1107. doi:10.1038/nn.3145

Thomson AM, West DC, Lodge D. 1985. An N-methylaspartate receptor-mediated synapse in rat cerebral cortex: a site of action of ketamine? Nature 313: 479-481.

Tian J, Uchida N. 2015. Habenula lesions reveal that multiple mechanisms underlie dopamine prediction errors. Neuron $\mathbf{8 7}$ : 1304-1316. doi:10.1016/j.neuron.2015.08.028

Warden MR, Selimbeyoglu A, Mirzabekov JJ, Lo M, Thompson KR, Kim SY, Adhikari A, Tye KM, Frank LM, Deisseroth K. 2012. A prefrontal cortex-brainstem neuronal projection that controls response to behavioural challenge. Nature 492: 428 432. doi:10.1038/nature11617
Weiss T, Veh RW. 2011. Morphological and electrophysiological characteristics of neurons within identified subnuclei of the lateral habenula in rat brain slices. Neuroscience 172: 74-93. doi:10.1016/j.neuroscience.2010.10.047

Wilcox KS, Gutnick MJ, Christoph GR. 1988. Electrophysiological properties of neurons in the lateral habenula nucleus: an in vitro study. J Neurophysiol 59: 212-225. doi:10.1152/ jn.1988.59.1.212

Wohleb ES, Franklin T, Iwata M, Duman RS. 2016. Integrating neuroimmune systems in the neurobiology of depression. Nat Rev Neurosci 17: 497-511. doi:10.1038/nrn.2016.69

Yang Y, Cui YH, Sang KN, Dong YY, Ni ZY, Ma SS, Hu HL. 2018a. Ketamine blocks bursting in the lateral habenula to rapidly relieve depression. Nature 554: 317-322. doi: 10.1038/nature25509

Yang Y, Wang H, Hu J, Hu H. 2018b. Lateral habenula in the pathophysiology of depression. Curr Opin Neurobiol 48: 90 96. doi:10.1016/j.conb.2017.10.024

Zanos P, Thompson SM, Duman RS, Zarate CA Jr, Gould TD. 2018. Convergent mechanisms underlying rapid antidepressant action. CNS Drugs 32: 197-227.

Zarate CA, Singh JB, Carlson PJ, Brutsche NE, Ameli R, Luckenbaugh DA, Charney DS, Manji HK. 2006. A randomized trial of an $\mathrm{N}$-methyl-D-aspartate antagonist in treatmentresistant major depression. Arch Gen Psychiat 63: 856-864.

Zarate CA, Brutsche NE, Ibrahim L, Franco-Chaves J, Diazgra$\operatorname{nados}$ N, Cravchik A, Selter J, Marquardt CA, Liberty V, Luckenbaugh DA. 2012. Replication of ketamine's antidepressant efficacy in bipolar depression: a randomized controlled add-on trial. Biol Psychiat 71: 939-946.

Zhu ZT, Munhall A, Shen KZ, Johnson SW. 2005. NMDA enhances a depolarization-activated inward current in subthalamic neurons. Neuropharmacology 49: 317-327. 


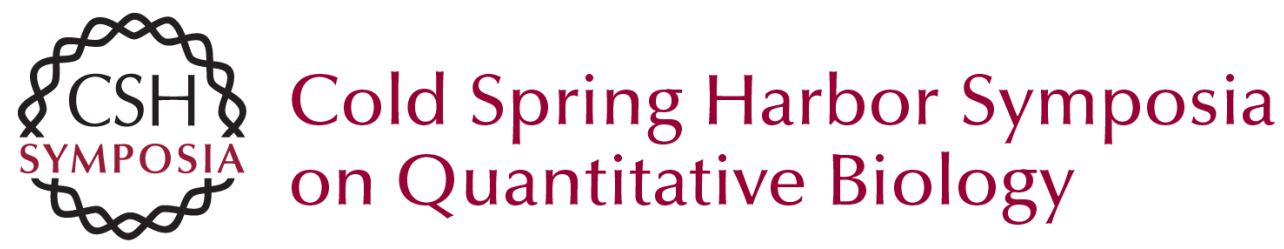

\section{Decoding Depression: Insights from Glial and Ketamine Regulation of Neuronal Burst Firing in Lateral Habenula}

Yihui Cui, Yan Yang, Yiyan Dong, et al.

Cold Spring Harb Symp Quant Biol 2018 83: 141-150 originally published online February 4, 2019

Access the most recent version at doi:10.1101/sqb.2018.83.036871

References This article cites 67 articles, 8 of which can be accessed free at: http://symposium.cshlp.org/content/83/141.full.html\#ref-list-1

Creative This article is distributed under the terms of the

Commons http://creativecommons.org/licenses/by-nc/4.0/, which permits reuse and

License redistribution, except for commercial purposes, provided that the original author and source are credited.

Email Alerting Receive free email alerts when new articles cite this article - sign up in Service the box at the top right corner of the article or click here. 